\title{
La Société internationale francophone d'éducation médicale (SIFEM) : 20 ans au service de la promotion de l'innovation, de la collaboration et de la recherche dans le champ de la formation des professionnels de la santé
}

Chères lectrices, chers lecteurs,

Vous trouverez, dans ce nouveau numéro de Pédagogie Médicale, des articles de recherche en éducation des sciences de la santé et trois tribunes que les auteurs des conférences plénières du Congrès international de pédagogie des sciences de la santé (CIFPSS) de mai 2021 ont accepté de retranscrire pour les partager avec vous. Ce numéro reflète la dynamique de recherche, la pluralité des thématiques abordées et la place centrale qu'occupe l'interprofessionnalité au sein de notre communauté. La rédaction de la revue a confié la rédaction de l'éditorial de ce numéro au bureau de la SIFEM, nous permettant de communiquer sur l'évolution de notre société savante, comme nous avons l'habitude de le faire régulièrement.

\section{Renouvellement du conseil d'administration de la SIFEM}

Les statuts de la SIFEM prévoient le renouvellement des membres du conseil d'administration (CA) par tiers tous les trois ans. L'appel à candidatures au printemps dernier a été fructueux et le vote des adhérents qui a suivi, par voie électronique, a permis de renouveler le CA dans le maintien de la représentation internationale et la pluralité des professions de santé.

Nous adressons tous nos remerciements à Elie Nemr (médecin hospitalo-universitaire à Beyrouth), membre sortant, pour son engagement au CA pendant de nombreuses années. Elie Nemr reste membre du comité scientifique de nos congrès. Nous saluons également Céline Monette (Forum des médecins francophones du Québec), membre sortante. Nous félicitons Marie-Claude Audétat (formatrice et chercheure en éducation des sciences de la santé à Genève), déjà membre et réélue. Le CA se renouvelle partiellement avec l'arrivée d'Alexandre Bellier (médecin hospitalo-universitaire à Grenoble) et d'Isabelle Sebri (cadre de santé aux Hôpitaux universitaires de Strasbourg). En accord avec les membres du conseil, Bernard Charlin, co-fondateur de la SIFEM, a souhaité mettre fin à ses fonctions de président qu'il assumait depuis 2017. Ainsi, à la suite de Jean-François Denef, Bernard Charlin reprend le rôle de «président sortant». Dans cette nouvelle fonction, il continuera à exercer au sein du bureau les prérogatives du président sortant, comme le prévoient les statuts. Jean-François Denef se consacre désormais au Comité d'éthique de la recherche de la SIFEM, dont il est le président. Le CA a nommé Anne Demeester présidente de la SIFEM; elle quitte ainsi la fonction de trésorière, qu'elle assurait depuis 2009 et qui est reprise par Isabelle Sebri. Thierry Pelaccia est nommé vice-président avec pour mission principale d'assurer la direction scientifique des événements organisés par la SIFEM. Etienne Panchout est nommé secrétaire général de la SIFEM et rejoint le bureau. La liste complète du conseil d'administration et la biographie de ses membres sont accessibles sur le site de la SIFEM (https://www. sifem.net/fr/organisation).

\section{Continuité des missions assurées auprès des membres de la SIFEM}

\section{La revue Pédagogie Médicale}

Chaque adhérent à la SIFEM bénéficie d'un abonnement électronique à la revue Pédagogie Médicale (https:/ / www.pedagogie-medicale.org/). Notre revue a plus de vingt ans! Le saviez-vous? Les premiers numéros de Pédagogie Médicale ont été publiés en 2000. La naissance de la revue a précédé celle de la SIFEM dont elle est devenue l'organe officiel d'expression. Au fil de 22 tomes et de 85 numéros, près de 500 articles originaux ont été publiés. La qualité et la pérennité de cette entreprise ont permis à la revue d'être accréditée en 2020 par le Haut Conseil de l'évaluation de la recherche et de l'enseignement supérieur (HCERES) et par le Conseil national des universités (CNU) dans la section des sciences de l'éducation, ce qui permet désormais à la fois aux équipes de recherche et aux auteurs concernés de valoriser leurs travaux, lors de leur recrutement et pour leur promotion dans une carrière universitaire. Cette reconnaissance témoigne du dynamisme des contributeurs, du processus de révision rigoureux assuré par les relecteurs experts et le comité de rédaction, sous la coordination de Jean Jouquan (rédacteur en chef) et de Nicolas Fernandez (rédacteur en chef adjoint). La prochaine étape, déjà amorcée, consistera à déposer le dossier d'évaluation en vue de l'indexation de 
la revue dans les bases de données internationales (en particulier Medline). Nous ne manquerons pas de vous informer de l'avancement de ce dossier complexe et exigeant.

\section{Les événements scientifiques et pédagogiques organisés par la SIFEM}

La dernière édition du CIFPSS s'est tenue à distance depuis Strasbourg, en mai 2021. Elle a réuni près de 400 participants, qui ont soumis 40 propositions d'atelier et 200 communications libres. Il s'agit d'un record qui témoigne du dynamisme de l'éducation des sciences de la santé et de la recherche dans ce champ en francophonie, et ce, malgré le contexte sanitaire. Le CIFPSS 2022 aura lieu $\mathrm{du} 1^{\mathrm{er}}$ au 3 juin à Amiens. L'appel à communications sera lancé au courant du mois de novembre. Toutes les informations seront prochainement accessibles sur le site de la manifestation (https://cifpss.org/fr/).

Sur le plan scientifique, la SIFEM organise également depuis un an des webinaires, les derniers mercredis de chaque mois. Après une série de rendez-vous consacrés au portfolio, Nicolas Picard et Maxime Ducret, chargés par le bureau de l'animation de ces réunions, ont choisi pour ce trimestre la thématique de l'évaluation des apprentissages, avec un focus particulier sur les examens cliniques objectifs structurés (ECOS); une nouvelle occasion d'inviter des experts à partager leur passion et leurs pratiques avec la communauté des membres de la SIFEM.

\section{Accompagnement de la recherche en éducation médicale}

Bourse de recherche

Dans le cadre du CIFPSS, la SIFEM finance deux bourses d'un montant de 2000 euros chacune, qui font l'objet d'un appel à candidatures auprès de ses membres adhérents. La première est destinée à accompagner un projet de recherche original dans le champ de l'éducation des sciences de la santé. La seconde a pour but d'aider les formateurs et les enseignants à concevoir et à déployer un dispositif original de formation des étudiants et/ou des professionnels de la santé.

\section{Comité d'éthique de la recherche}

L'approbation éthique préalable des projets de recherche est une exigence désormais formulée par les comités de rédaction des revues internationales lors de la soumission d'articles. Il existe donc un réel besoin pour le domaine de l'éducation des professions de santé. Pour y répondre, la SIFEM a institué un comité d'évaluation de l'intégrité et de l'éthique des projets de recherche (https://www.sifem.net/ fr/comite-ethique-recherche), dont l'avis peut être sollicité par les porteurs de projets de recherche, dès lors qu'ils sont membres de la SIFEM.

Le CA et le bureau de la SIFEM s'engagent à poursuivre le développement de notre société savante au service de la mission principale définie par ses fondateurs : «promouvoir la santé dans le monde francophone par la formation des professionnels et la recherche en éducation». Nous restons attentifs à vos suggestions pour faire évoluer nos activités scientifiques et pédagogiques, et répondre à vos nouvelles attentes. N'hésitez pas à nous écrire pour partager vos idées et besoins!

$$
\begin{array}{r}
\text { Anne DEMEESTER, Thierry PELACCIA, } \\
\text { Bernard CHARLIN } \\
\text { Présidente, Vice-président, Président sortant } \\
\text { https://www.sifem.net/fr/ }
\end{array}
$$

